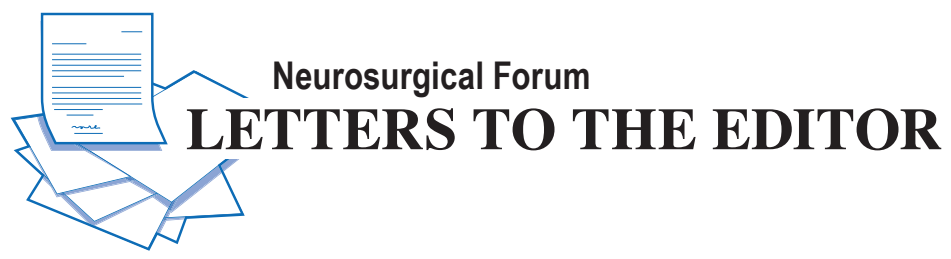

\section{Surgery for pediatric thalamic tumors: using DTI to improve neurological outcome}

TO THE EDITOR: We read with great interest the article published by Cinalli et al. ${ }^{2}$ (Cinalli G, Aguirre DT, Mirone G, et al: Surgical treatment of thalamic tumors in children. J Neurosurg Pediatr 21:247-257, March 2018). The authors present a series of 25 children who underwent operations for thalamic tumors (9 patients) and thalamopeduncular tumors (16 patients) from 2002 to 2016. Different surgical approaches (anterior interhemispheric, posterior interhemispheric, and subtemporal) were chosen according to tumor location. Gross-total resection (GTR) was achieved in 15 patients $(60 \%)$, subtotal resection in $4(16 \%)$, and partial resection in $6(24 \%)$.

The authors attribute accurate preoperative surgical planning as the key element that allows radical tumor resection while keeping surgical morbidity at acceptable levels. They also commented on the utility of diffusion tensor imaging (DTI) to identify the safest surgical corridor for thalamic tumors (in 16 of 25 cases to locate the corticospinal tracts [CSTs]). Their series had a surgical morbidity rate of $48 \%$ and mortality rate of $8 \%$. It would be interesting to know the difference in terms of neurological outcome between the patients operated on using DTI-based planning and the rest of the series. Furthermore, the authors did not specify how preoperative DTI influenced the selection of the surgical approach.

We prefer to use DTI-based surgical planning for all tumors with a risk of damage to eloquent structures during tumor removal. In fact, these acquisitions can also be performed for surgical planning even in children younger than 2 years of age. ${ }^{1}$ We would like to illustrate the case of a 14-month-old child who was admitted for a severe hemiparesis (M2/M5 according to the Oxford scale) due to a thalamic tumor (Fig. 1A and B).

To choose the ideal surgical corridors among frontal transcortical, interhemispheric, and transparietal transcortical approaches, we performed DTI tractography. Due to the displacement of the CST anterior and medial to the tumor (Fig. 1C and D), we opted for the transcortical superior parietal lobule approach. Continuous somatosensory evoked potential monitoring along with intermittent white matter motor stimulation was used throughout the resection.

The patient's motor function improved, with strength of the right side of the body at M3/5. Postoperative MRI confirmed the GTR of the lesion (Fig. 2A and B). DTI reconstruction showed the preservation of the CST (Fig. 2C and D). The histopathological examination revealed an atypical rhabdoid-teratoid tumor (WHO grade IV). The patient was included in the phase III protocol (FDA no. ACNS00333; clinicaltrials.gov no. NCT00653068) and received high-dose induction chemotherapy followed by stem cell rescue treatment and consolidation chemotherapy according to the protocol guidelines. The follow-up MRI at 3 months after surgery showed no tumor recurrence.

The present case supports the use of DTI tractogra-
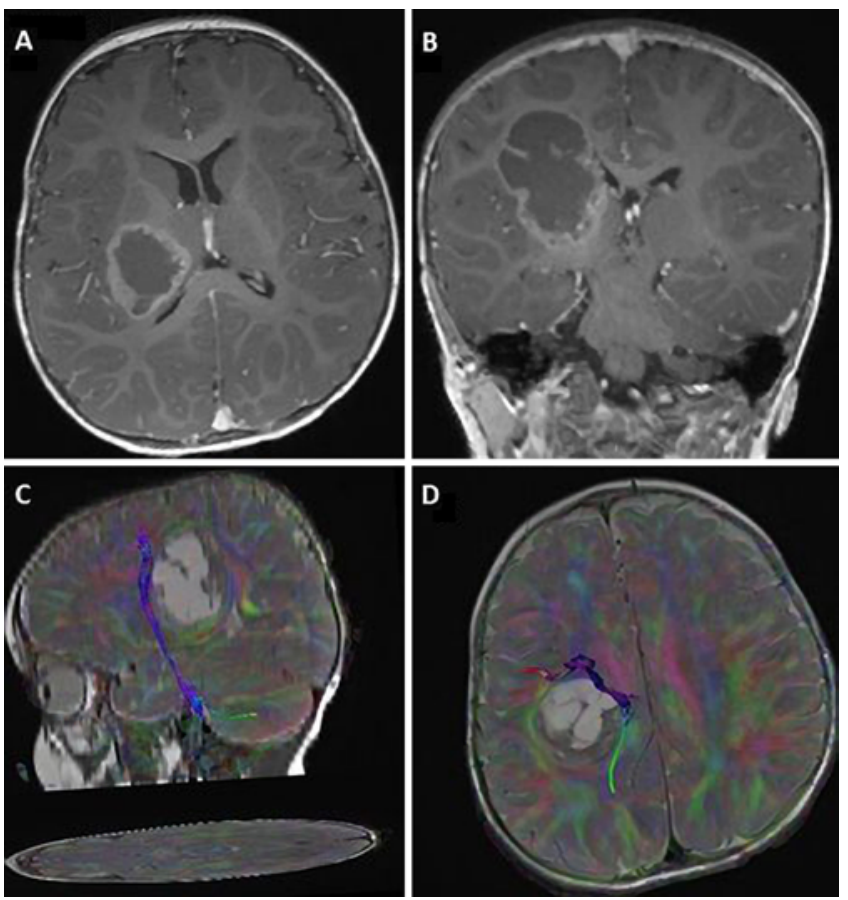

FIG. 1. Preoperative MRI. Axial (A) and coronal (B) sequences showing a right thalamic tumor. Sagittal (C) and axial (D) DTI sequences showing the CST, displaced anteromedially to the thalamic tumor. 

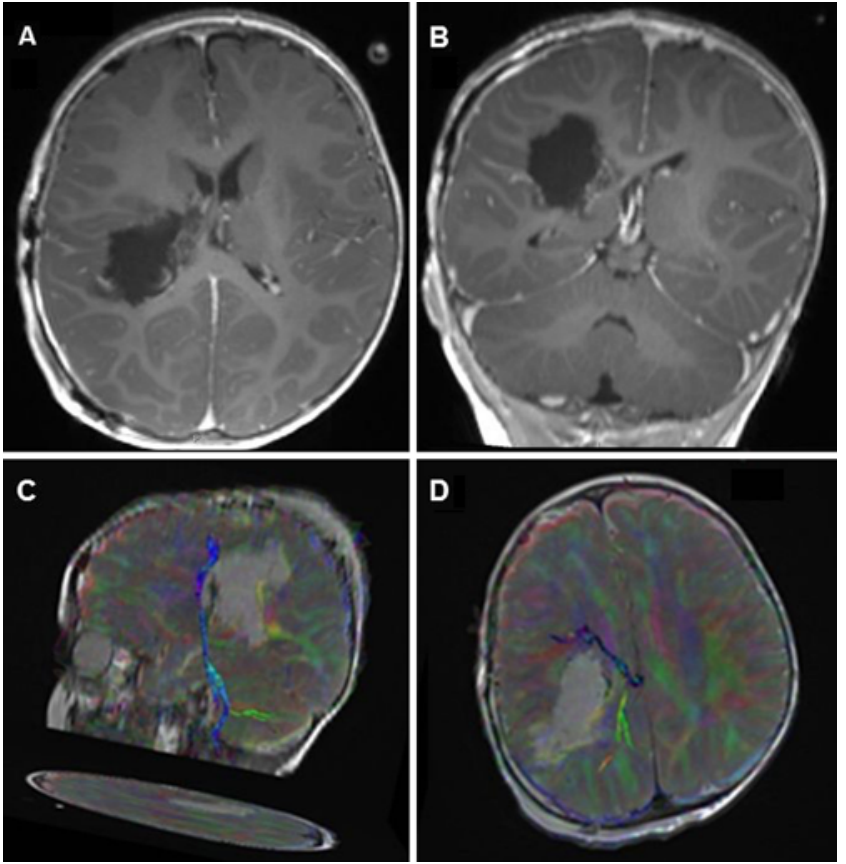

FIG. 2. Postoperative MRI. Axial (A) and coronal (B) T1-weighted enhanced sequences showing complete tumor removal. Sagittal $(\mathbf{C})$ and axial (D) DTI sequences showing CST preservation.

phy for resection of thalamic tumors even in very young children $(<2$ years old). As confirmed by recent studies by Braga et al., ${ }^{1}$ DTI can define the anatomy of the CST with acceptable accuracy, even in very young children, due to the precocious myelination of the CST (around 34 gestational weeks) compared to other white matter tracts. The principal limitation of cerebral DTI in young children is the underestimation of CST volumes due to the limited brain volume and superposition of same-size voxels. ${ }^{3}$ This technical problem of DTI acquisition can be addressed by the use of smaller voxel volumes as performed in our patient. In conclusion, we strongly suggest the use of cerebral DTI as a routine assessment for children with pediatric thalamic tumors, even in very young patients.

\section{Rodolfo Maduri, MD ${ }^{1}$ Viviana Aureli, MD1 Vincent Dunet, MD1 Roy Thomas Daniel, $\mathrm{MCh}^{1,2}$ Mahmoud Messerer, MD ${ }^{1,2}$ ${ }^{1}$ Lausanne University Hospital (CHUV), Lausanne, Switzerland ¿University of Lausanne (UniL), Lausanne, Switzerland}

\section{Acknowledgments}

We heartily thank Dr. Manuel Diezi (Pediatric Unit of Hematology and Oncology, Department of Pediatric Medicine and Surgery, CHUV) for his qualified collaboration.

\section{References}

1. Braga RM, Roze E, Ball G, Merchant N, Tusor N, Arichi T, et al: Development of the corticospinal and callosal tracts from extremely premature birth up to 2 years of age. PLoS One 10:e 0125681,2015

2. Cinalli G, Aguirre DT, Mirone G, Ruggiero C, Cascone D, Quaglietta L, et al: Surgical treatment of thalamic tumors in children. J Neurosurg Pediatr 21:247-257, 2018

3. Jeong JW, Lee J, Kamson DO, Chugani HT, Juhász C: Detection of hand and leg motor tract injury using novel diffusion tensor MRI tractography in children with central motor dysfunction. Magn Reson Imaging 33:895-902, 2015

\section{Disclosures}

The authors report no conflict of interest.

\section{Correspondence}

Rodolfo Maduri: rodolfo.maduri@gmail.com.

INCLUDE WHEN CITING

Published online September 7, 2018; DOI: 10.3171/2018.5.PEDS18244.

\section{Response}

We thank Dr. Maduri and coworkers for their interest in our paper. We did not compare neurological outcome before and after the use of DTI. In a retrospective series covering 14 years and only 25 cases it would have been very unfair to propose this kind of analysis, especially because before the DTI era, as written in the Methods, standard MRI in T2-weighted and FLAIR sequences was already able to offer excellent information about internal capsule displacement/infiltration, always allowing surgical trajectories that were able to spare the CST. Nevertheless, the impact of DTI cannot be underestimated. The possibility to visualize the CST on every single slice of a volumetric sequence and study the 3D relationship between the tumor and all the main bundles of white matter fibers (not only the CST, but also optic radiations, etc.) offers extraordinary possibilities to the surgeon to select the best surgical trajectory. This aspect, although quite intuitive, was extensively explained in the paragraph "Surgical Approach," and practical examples were also included in the 4 videos that I warmly invite Dr. Maduri to view for a better understanding. In any case, DTI was not the only tool used to decide the surgical corridor. This corridor is selected on the basis of several factors such as tumor dimensions and shape; direction of the longest tumor axis; presence of a peduncular component; presence, position, and dimensions of the tumor cyst; severity of hydrocephalus; and relation with the ventricles, as extensively explained in the description of surgical techniques. We would like to congratulate Dr. Maduri and his team for the beautiful images and the excellent surgical and clinical result in their case. The first important observation is that age is not a limit for DTI reconstruction in the hands of dedicated neuroradiologists with pediatric experience; we had the same results in very small babies with reliable reconstructions. Moreover, their case helps to demonstrate that careful preoperative planning, 3D reconstruction of the DTI model of the CST, and mastering a high-quality microneurosurgical technique can maximize the surgical result of patients with thalamic tumors, simplify the treatment, offer the highest overall survival and progression- 
free survival perspectives in aggressive histologies as in their case or, in the case of low-grade tumors, reduce the use of adjuvant therapies (chemo-/radiotherapy) that probably appear less invasive in the very short term but carry mid- and long-term consequences.

Giuseppe Cinalli, MD Lucia Quaglietta, MD, PhD Santobono-Pausilipon Children's Hospital, Naples, Italy

INCLUDE WHEN CITING

Published online September 7, 2018; DOI: 10.3171/2018.7.PEDS18306.

CAANS 2018, except where prohibited by US copyright law

\section{Reappraisal of nuchal ligament as a dura substitute for Chiari malformation type I}

TO THE EDITOR: We read with interest the article by Cools et al. ${ }^{1}$ (Cools MJ, Quinsey CS, Elton SW: Chiari decompression outcomes using ligamentum nuchae harvest and duraplasty in pediatric patients with Chiari malformation type I. J Neurosurg Pediatr 22:47-51, July 2018). As the authors note, the choice of material to be used for duraplasty around the herniated tonsil remains a matter of debate. In their study, they re-appraised Kosnik's method, which is to harvest the nuchal ligament for a patch graft. ${ }^{2}$ Cools and colleagues reported quite good results for patients with symptomatic Chiari malformation type I, but we would like to know more about the 9 asymptomatic cases. Specifically, we wonder about the surgical indications in those asymptomatic cases and whether the nuchal ligament patch graft was effective for those patients.

In addition, the authors report that in 8 of the 19 cases in which a syrinx was present preoperatively, the syrinx was stable after the operation, and in 1 case the syrinx was described as "worsened." In the 16 cases in which the patients are described as having preoperative symptoms, symptoms are described as "stable" postoperatively in 1 case and "worsened" in 1 case. We would like to know what they consider to be the cause of lack of improvement in these cases. Was it due to the innate nature of the asymptomatic cases? Or was it related to shortcomings of the surgical method, which appears to not involve direct manipulation of the cerebellar tonsils (routinely performed by some surgeons in order to make a larger conduit for CSF)? Nevertheless, we congratulate the authors for drawing attention to the simple, less invasive, and less expensive autologous graft again, with minimal surgical complications.

Toshikazu Kimura, PhD

Shunsuke Ichi, MD

Japanese Red Cross Medical Center, Tokyo, Japan

\section{References}

1. Cools MJ, Quinsey CS, Elton SW: Chiari decompression outcomes using ligamentum nuchae harvest and duraplasty in pediatric patients with Chiari malformation type I. J Neurosurg Pediatr 22:47-51, 2018

2. Kosnik EJ: Use of ligamentum nuchae graft for dural closure in posterior fossa surgery. Technical note. J Neurosurg 89:155-156, 1998

\section{Disclosures}

The authors report no conflict of interest.

\section{Correspondence}

Toshikazu Kimura: tkim-tky@umin.ac.jp.

INCLUDE WHEN CITING

Published online September 7, 2018; DOI: 10.3171/2018.7.PEDS18403.

\section{Response}

No response was received from the authors of the original article.

CAANS 2018, except where prohibited by US copyright law 